\title{
Influência da aplicação de sistemas adesivos universais sob corrente elétrica nos valores de resistência de união à dentina
}

\author{
Gustavo Belmiro Casaburi, Mauricio Guarda, Marcus \\ Vinícius Loureiro Bertolo, Mário Alexandre Coelho Sinhoreti
}

\section{Resumo}

O objetivo deste estudo foi avaliar se a aplicação de um sistema adesivo universal aplicado tanto na forma convencional, quanto na autocondicionante na presença de corrente elétrica interfere na resistência de união ao dente. Para isso, foram utilizados 36 dentes bovinos divididos em 4 grupos: Grupo 1: Single Bond Universal + aplicação sem corrente elétrica + técnica condicionamento total; grupo 2: Single Bond Universal + aplicação sem corrente elétrica + técnica autocondicionante; grupo 3: Single Bond Universal + aplicação com corrente elétrica + técnica condicionamento total; grupo 4: Single Bond Universal + aplicação com corrente elétrica + técnica autocondicionante $(n=8)$. OS dentes foram cortados e os sistemas adesivos aplicados conforme os grupos de teste, sendo que naqueles onde há corrente elétrica, foi acoplado um dispositivo ao microbrush para garantir a passagem da corrente elétrica. Após a restauração, os dentes foram armazenados por 24 horas e após, cortados em palitos de $1 \mathrm{~mm}^{2}$. Então levados para teste de microtração para determinação da resistência de união. Os dados foram submetidos à análise estatística usando ANOVA dois fatores (protocolo de aplicação de corrente elétrica e técnica de aplicação) e as médias comparadas pelo teste de Tukey com nível de significância de 5\%. Foi concluído que no período de 24 horas não houve diferença significativa, a aplicação da corrente elétrica não resultou em um aumento significativo de resistência de união.

Palavras-chave: sistemas adesivos, corrente elétrica, resistência de união..

\section{Introdução}

A aplicação dos sistemas adesivos sob corrente elétrica pode ser considerada eficaz, pois aumenta os valores de resistência de união e não acarreta em um aumento do número de passos clínicos. Assim, seria interessante saber o comportamento de sistemas adesivos universais, de forma convencional ou autocondicionante quando aplicados sob corrente elétrica, já que ambos os procedimentos visam melhorar a adesão sem necessariamente aumentar o número de etapas clínicas e o tempo de atendimento.

\section{Resultados e Discussão}

Para a análise da resistência de união foram utilizados 32 incisivos bovinos, os dentes foram distribuídos em 4 grupos $(n=8)$, os quais foram cortados expondo a dentina, as superfícies lisas foram polidas para padronizar o smear layer e a aplicação dos sistemas adesivos foi realizada utilizando-se microbrush da forma convencional. Já a aplicação do sistema adesivo dos grupos experimentais realizada pela utilização de um microbrush acoplado a um dispositivo gerador de corrente elétrica desenvolvido no laboratório. Todos os dentes foram restaurados com compósito restaurador. Após o período de armazenamento, os palitos foram levados a uma máquina de ensaio de tração. Os valores obtidos com cada método de aplicação foram transformados em MPa e submetidos à análise estatística usando ANOVA dois fatores (protocolo de aplicação de corrente elétrica e técnica de aplicação) e as médias comparadas pelo teste de Tukey com nível de significância de $5 \%$.

Tabela 1: Média dos resultados de resistência de união após 24 horas de armazenamento.

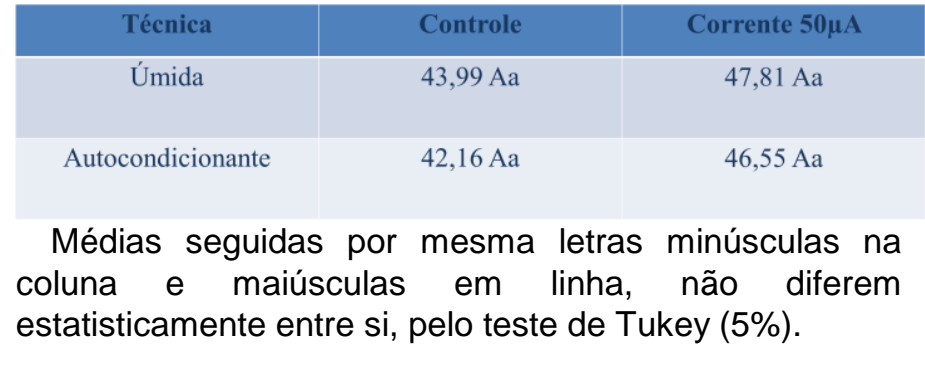

\section{Conclusões}

Pode-se observar que no período de 24 horas não houve diferença significativa entre a técnica utilizada para a confecção da restauração. A aplicação da corrente elétrica não resultou em um aumento significativo de resistência de união.

\section{Agradecimentos}

Faculdade de Odontologia de Piracicaba - Unicamp

\footnotetext{
1. Alex G. Universal adhesives: the next evolution in adhesive dentistry? Compend Contin Educ Dent. 2015;36(1):15-26

2. Yoshida Y, Inoue S. Chemical analyses in dental adhesive technology. Japanese Dental Science Review. 2012;48(2):141-52.

3. Muñoz MA, Luque I, Hass V, Reis A, Loguercio AD, Bombarda NHC. Immediate bonding properties of universal adhesives to dentine. J Dent. 2013;41(5):404-11.

4. Sano H, Shono T, Takatsu T, Hosoda H. Microporous dentin zone beneath resinimpregnated layer. Oper Dent. 1994; 19(2):59-64

5. Borges B, Souza-Junior E, Brandt W, Loguercio A, Montes M, Puppin-Rontani R et al. Degree of conversion of simplified contemporary adhesive systems as influenced by extended air-activated or passive solvent volatilization modes. Oper Dent. 2012; 37(3):246-52

6. Ito S, Hashimoto M, Wadgaonkar B, Svizero N, Carvalho RM, Yiu C et al. Effects of resin hydrophilicity on water sorption and changes in modulus of elasticity. Biomaterials. 2005; 26(33):6449-59.

7. Van Meerbeek B, Van Landuyt K, De Munck J, Hashimoto M, Peumans M, Lambrechts P et al. Technique-sensitivity of contemporary adhesives. Dent Mater J. 2005; 24(1):1-13.

8. Tay FR, Pashley DH, Suh B, Carvalho R, Miller M. Single-step, self-etch adhesives behave as permeable membrane after polymerization. Part I. Bond strength and morphologic evidence. Am J Dent. 2004; 17(4):271-8.

9. Pasquantonio G, Tay FR, Mazzoni A, Suppa P, Ruggeri A, Falconi M et al. Electric device improves bonds of simplified etch-and-rinse adhesives. Dent Mater. 2007; 23(4):513-8
} 